\title{
El impacto de la reforma educativa en la planeación de la práctica profesional
}

\section{The impact of educational reform in the planning of professional practice}

\author{
PONCE-CONTRERAS, María Guadalupe, COVARRUBIAS-SOLÍS Irma Fabiola, PÉREZ-BRIONES, \\ Nancy Griselda, TELLO-GARCÍA, María Ascención y NUNCIO-DOMÍNGUEZ, José Luis
}

Universidad Autónoma de Coahuila. Facultad de Enfermería, Dr. Santiago Valdés Galindo. Código postal 25600

ID $1^{\text {er }}$ Autor: María Guadalupe, Ponce-Contreras / CVU CONACYT ID: 94777

ID $1^{\text {er }}$ Coautor: Irma Fabiola, Covarrubias-Solís

ID $2^{\text {do }}$ Coautor: Nancy Griselda, Pérez-Briones

ID $3^{\text {er }}$ Coautor: María Ascención, Tello-García

ID $4{ }^{\text {to }}$ Coautor: José Luis, Nuncio-Domínguez / CVU CONACYT ID: 329596

DOI: $10.35429 / J U P .2019 .9 .3 .11 .24$

Recibido: 18 de Julio, 2019; Aceptado 26 de Septiembre, 2019

\section{Resumen}

La investigación se llevó a cabo en dos escuelas de educación formadoras de futuros educadores de la Escuela Normal de Educación Preescolar y la Benemérita Escuela Normal de Coahuila. Se pretende conocer el impacto de la Reforma Educativa en la planeación de la práctica profesional. La muestra fue de 70 sujetos. El instrumento tiene 4 variables signalíticas: edad, genero, escuela y especialidad, y 3 ejes: reforma, planeación y práctica, con veinte variables cada uno, la escala fue de Likert decimal. Se procesaron los datos en paquetes estadísticos, utilizando medidas de tendencia central y variabilidad, frecuencias y porcentajes, análisis integracional. Resultados. Se obtuvo un Alpha de Cronbach de .94. En el integracional, con la Reforma Educativa se pretende que los resultados del aprendizaje se relacionen con evaluaciones internacionales, así como también se pretende el aumento de la matrícula en educación básica de calidad y así puedan tener un máximo logro educativo. En el de regresión: los alumnos tienen un mejor desarrollo académico cuando los maestros llevan a cabo un diagnóstico del aprendizaje y se da una interacción positiva entre alumnomaestro, y que los padres aporten una opinión acerca de los programas educativos de sus hijos para que así en conjunto toda la comunidad se dé una educación de calidad.

Reforma educativa, Planeación, Práctica profesional

\begin{abstract}
The research was carried out in two higher education schools that train future educators of the Normal School of Preschool Education and the Benemérita Normal School of Coahuila. It is intended to know the impact of the Educational Reform in the planning of professional practice. The sample was 70 subjects. The instrument has 4 signaling variables: age, gender, school and specialty, and 3 axes: reform, planning and practice, with twenty variables each, the scale was decimal Likert. Data were processed in statistical packages, using measures of central tendency and variability, frequencies and percentages, integrative analysis. Results A Cronbach Alpha of .94 was obtained. In the integrative one, with the Educational Reform it is intended that the learning outcomes be related to international assessments, as well as the increase in enrollment in quality basic education and so that they can have a maximum educational achievement. In the regression: students have a better academic development when teachers carry out a diagnosis of learning and there is a positive interaction between student-teacher, and that parents provide an opinion about their children's educational programs so that so as a whole the whole community is given a quality education.
\end{abstract}

Educational reform, Planning, Professional practice

Citación: PONCE-CONTRERAS, María Guadalupe, COVARRUBIAS-SOLÍS Irma Fabiola, PÉREZ-BRIONES, Nancy Griselda, TELLO-GARCÍA, María Ascención y NUNCIO-DOMÍNGUEZ, José Luis. El impacto de la reforma educativa en la planeación de la práctica profesional. Revista de Políticas Universitarias. 2019. 3-9: 11-24.

\footnotetext{
* Correspondencia al Autor (Correo electrónico: mariaponce @uadec.edu.mx)

$\dagger$ Investigador contribuyendo como primer autor.
} 


\section{Introducción}

La planeación ofrece un marco metodológico que puede encontrar una aplicación en la estructuración y conformación de un currículo. El uso de elementos conceptuales de la planeación estratégica en el diseño curricular facilita la organización de la información, involucrada en el proceso de conformación de un currículo educación superior.

La forma sistemática de organizar de modo integral el currículo es la planeación. Ésta se definiría, aplicada al diseño curricular, como el proceso mediante el cual se identifica la razón de ser (o del currículo, se vislumbran su estructura desarrollo tanto actual como proyectado en tiempo, y se establecen los objetivos, metas y acciones considerados necesarios para alcanzar el nivel proyectado y los resultados del currículo. (Devalle de Rendo A. (2010).

En términos generales, la planeación es el proceso estructurado por el cual una organización social — tal como una institución educativa- define su razón de ser en el entorno donde realiza sus actividades, vislumbra su estado deseado en el futuro, desarrolla los objetivos y las acciones concretas para llegar a alcanzar el mencionado estado deseado. El propósito global de la planeación es tener una visión clara de hacia dónde dirigir los recursos y las acciones, al tiempo mantiene la flexibilidad necesaria para incorporar factores indeterminables o inciertos del entorno, la probabilidad de conseguir el estado que se busca en la organización social y en el propio entorno. Op.Cit (2010).

La investigación se llevó a cabo en dos escuelas de educación superior formadoras de futuros educadores es decir en la Escuela Normal de Educación Preescolar y la Benemérita Escuela Normal de Coahuila, esta ultima de educación primaria. En la presente investigación se pretende conocer el impacto de la Reforma Educativa en la planeación de la práctica profesional, es decir como los nuevos lineamientos de la Reforma Educativa modificaron la forma de planear para realizar la práctica profesional.

\section{Antecedentes}

El diseño curricular es un proceso que deben efectuar las instituciones de educación superior con cierta frecuencia a fin de ampliar o actualizar su oferta educativa. El resultado del diseño curricular es el documento donde se explicitan organización, operación y evaluación integral de la formación propuesta, esto el currículo. La fase de planeación en el diseño curricular es de suma importancia porque de ésta depende la correcta operación, o en todo caso la operación riesgos de error, y la evaluación adecuada del currículo. La planeación ofrece un marco metodológico que puede encontrar una aplicación en la estructuración y conformación de un currículo. El uso de elementos conceptuales de la planeación estratégica en el diseño curricular facilita la organización de la información, involucrada en el proceso de conformación de un currículo educación superior.

La fase más importante del diseño curricular es la planeación, esto es el planteamiento teórico y fundamentado de las actividades que propicien los resultados esperados del currículo. Una forma sistemática de organizar de modo integral el currículo es la planeación. Ésta se definiría, aplicada al diseño curricular, como el proceso mediante el cual se identifica la razón de ser (o del currículo, se vislumbran su estructura desarrollo tanto actual como proyectado en tiempo, y se establecen los objetivos, metas y acciones considerados necesarios para alcanzar el nivel proyectado y los resultados del currículo. (Devalle de Rendo A. (2010).

En términos generales, la planeación es el proceso estructurado por el cual una organización social — tal como una institución educativa- define su razón de ser en el entorno donde realiza sus actividades, vislumbra su estado deseado en el futuro, desarrolla los objetivos y las acciones concretas para llegar a alcanzar el mencionado estado deseado. El propósito global de la planeación es tener una visión clara de hacia dónde dirigir los recursos y las acciones, al tiempo mantiene la flexibilidad necesaria para incorporar factores indeterminables o inciertos del entorno, la probabilidad de conseguir el estado que se busca en la organización social y en el propio entorno. Los principales elementos de la planeación estratégica son (Devalle de Rendo A. (2010). 


\section{El currículo es el resultado de:}

a) El análisis y reflexión sobre las características del contexto, del educando y de los recursos

b) La definición (tanto implícita y explícita) de los fines y los objetivos educativos.

c) La especificación de los medios y los procedimientos propuestos para asignar racionalmente los recursos humanos, materiales, informativos, financieros, temporales y organizativos de manera tal que se logren los fines propuestos (Ander-Egg, Ezequiel. 2004).

La integración de un currículo que incluya esos aspectos esenciales sólo se consigue mediante la organización sistemática de la información disponible para elaborarlo. La aplicación de los elementos de planeación estratégica posibilita un diseño curricular apropiado en particular para los propósitos la educación superior antes indicados.

Aunque la planeación propone como punto inicial la formulación de la misión del currículo, esto sólo es posible si se realiza un análisis preliminar, reuniendo la mayor cantidad de información posible, para identificar las necesidades sociales, en especial - pero no exclusivo - en el mercado laboral, que puedan ser potencialmente cubiertas por un nuevo plan de educación superior o por reestructuración de uno existente. Como resultado de este diagnóstico se justificaría, en principio, arranque del proceso de diseño curricular.

Puesto que la fase operativa del currículo tendrá lugar en efecto en una institución educativa, la del currículo deberá ser elaborada evitando contraposición con la misión de la institución, de forma se incurriría en un conflicto de intereses. Determinar la misión del currículo debe ser considerado dos componentes básicos:

a) El análisis sectorial, conformado por la identificación de necesidades sociales donde se dé la inserción de individuos con ciertos conocimientos, habilidades y actitudes; por análisis de la estructura de otros desarrollos curriculares similares existentes en el mundo por las tendencias generales en los campos conocimiento implicados en el currículo. b) Los modelos de desarrollo cognitivo, de aprendizaje y pedagógicos que permitirán sustentar al currículo en lo que respecta a la estructuración de los programas específicos de las unidades de aprendizaje o cursos que se incluyan él, así como del mapa curricular.

Con esta información esencial es posible, en principio, definir la misión y delinear el perfil de egreso currículo. Incluso podría afirmarse que la misión del currículo consiste fundamentalmente en disponer todos los factores necesarios para la consecución perfil de egreso. En el perfil de egreso se plasman los resultados esperados del currículo en una forma concreta.

Con independencia del currículo específico bajo estudio, se han marcado diversas características generales deseables en las personas que concluyen estudios en una institución de educación superior (Lafrancesco, Giovanni 2010).

Las tendencias marcan que los egresados de un mapa curricular de educación superior posean conocimientos profundos de los principios científicos esenciales sobre los que se sustente su formación específica, complementados con avances recientes que definan la actualización del currículo. En virtud de la alta velocidad de generación de conocimientos que ha caracterizado a las últimas décadas, no resulta ser una buena estrategia tratar de incluir en el currículo los últimos descubrimientos científicos o avances tecnológicos por el riesgo de obsolescencia inmediata.

Por el contrario, es preferible el estudio de los principios básicos que facilitarán la rápida asimilación de conocimientos nuevos, estableciendo así la necesidad de continuar la formación a lo largo del desarrollo profesional del individuo, pero no centrándose en un currículo de educación superior.

Por otro lado, el conjunto de conocimientos contemplados en el currículo no debería restringirse a los conocimientos técnicos del área, porque se requiere desarrollar en los individuos una sensibilización al seguimiento de sucesos sociales y económicos contemporáneos. 
La asimilación de conocimientos esenciales a los que se refiere el currículo está en función, al menos parcialmente, del dominio de las tecnologías informatizadas de búsqueda de datos. En efecto, otra habilidad fundamental derivada de cualquier currículo de educación superior es el uso de equipos tecnologías de computación para la búsqueda de y para otras aplicaciones específicas. El dominio de los procesos de comunicación oral y escrita, son habilidades indispensables que corresponden a resultados deseables del currículo y, en esa condición, deberán formar parte del perfil de egreso. Además de propiciar la asimilación de los conocimientos esenciales en el área de atribución del currículo, se debe promover lo largo de la estructura curricular la de integrar conocimientos, o genérica, con el propósito de resolver problemas. Esto significa que el énfasis de los programas aprendizaje incluidos en el currículo debe estar en impulsar el desarrollo cognitivo del individuo, introduciendo contenidos para estimulación del razonamiento lógico y para el logro del aprendizaje. De esta forma, se al perfil de egreso una profunda capacidad de análisis, integración e de información.

Es medular anotar en el currículo el reconocimiento de la importancia que tiene la interacción con otros seres humanos entorno inmediato o de otras culturas, puesto que tiende a inducir las de tolerancia respeto necesarias para la incorporación la organización de equipos de trabajo multidisciplinario en continuo cambio requiere habilidades de interpretación entorno sumamente desarrolladas.

El fomento de otras actitudes tales como el reconocimiento de responsabilidades éticas y sociales, también importante para su inclusión en el currículo y, bajo ciertas consideraciones, en el perfil de egreso.

\section{Planteamiento de investigación}

En este apartado se dará a conocer el planteamiento de la presente investigación, así como el contexto en el que será insertada. Otro tema importante dentro de este apartado será el conocer la interrogante de investigación, los objetivos que se plantearon con la misma, así como la justificación del porque querer investigar sobre el tema. Se conocerá también el contexto en que la presente investigación será realizada.

\section{Campo o área del saber.}

La línea de investigación se centra en los procesos de enseñanza-aprendizaje en el Sistema Educativo esto conlleva a estudios, reflexiones e investigaciones sobre proyectos educativos que caracterizan las etapas del acto educativo. Enseñanza y aprendizaje forman parte de proceso que tiene como fin la formación del estudiante.

El término enseñar se puede conceptualizar como señalar algo a alguien, es decir no enseñar cualquier cosa sino mostrar lo que se desconoce. Hernández, P. (2015). Esto implica que hay un sujeto que conoce, se puede decir que es el que puede enseñar, y otro que desconoce el que puede aprender. El que puede, quiere y sabe enseñar es el maestro y el que puede, quiere y sabe aprender es el alumno entonces debe de existir disposición por parte de los agentes de la educación, es decir el maestro y el alumno.

Aparte de estos agentes, están los contenidos, esto es, lo que se quiere enseñar o aprender como los programas curriculares y los procedimientos o instrumentos para enseñarlos o aprenderlos estos son los medios por los cuales se imparte la educación. Cuando se enseña algo es para conseguir alguna meta planteada como objetivo. Por otro lado, el acto de enseñar y aprender se da a partir de ciertas condiciones físicas, sociales y culturales conocidas como contexto escolar.

\section{Definición de la investigación}

En la presente investigación se busca que los estudiantes de las escuelas normales de educación manifiesten cuál consideran cual es impacto de la reforma educativa en la planeación de la práctica docente, esto dependerá de aspectos diversos como lo son el análisis de la planeación, los objetivos de la misma, el campo laboral, entre otras cuestiones. Cabe señalar que esto se reflejará a través de una explicación descriptiva de frecuencias y porcentajes, un análisis integracional, factorial y de regresión con base a una técnica de correlación de variables.

\section{Interrogantes Básicas}

¿Con la reforma educativa, se mejorará la calidad educativa?

PONCE-CONTRERAS, María Guadalupe, COVARRUBIAS-SOLÍS Irma Fabiola, PÉREZ-BRIONES, Nancy Griselda, TELLO-GARCÍA, María Ascención y NUNCIO-DOMÍNGUEZ, José Luis. El impacto de la reforma educativa en la planeación de la práctica profesional. Revista de Políticas Universitarias. 2019 
¿Se busca una innovación educativa, por medio de dicha Reforma?

¿Los objetivos de la planeación docente cambian debido a la Reforma Educativa?

¿Existen nuevas estrategias didácticas que sustenten un nuevo diseño de planeación?

¿La práctica profesional es parte de una función pedagógica donde se busca el mayor aprovechamiento académico de los estudiantes?

¿Los nuevos docentes tienen las suficientes herramientas para la resolución de problemas dentro del aula?

\section{Justificación}

La presente investigación es importante tanto para el investigador, los egresados y como para la sociedad en general, ya que la educación es un tema que siempre está a la vanguardia y en la actualidad el contar con una buena preparación académica la cual abre las puertas a un mejor trabajo y un mejor salario y por ende una mejor calidad de vida. Se trabajará con estudiantes de escuelas normales de educación superior de especialidades en preescolar y primaria donde se puedan distinguir las diferentes expectativas, experiencias y actitudes; por ello el presente trabajo de investigación pretende que los estudiantes normalistas expresen cuales son los significados que tienen acerca de la reforma educativa y como esta impacta en la planeación de la práctica docente.

\section{Objetivo general}

Conocer el impacto que tiene la Reforma Educativa en la planeación de la práctica profesional.

\section{Objetivos específicos}

Analizar la reforma educativa en cuanto a la mejora de la calidad educativa

Descubrir si existe una innovación educativa, por medio de dicha Reforma.

Relacionar los objetivos de la planeación con el cambio en la Reforma Educativa.

Descubrir nuevas estrategias didácticas que sustenten un nuevo diseño de planeación.
Detectar si la práctica profesional es parte de una función pedagógica donde se busca el mayor aprovechamiento académico de los estudiantes.

Analizar si los nuevos docentes cuentan con las suficientes herramientas para la resolución de problemas dentro del aula.

\section{Contexto donde pretende insertarse}

Se optó por buscar estudiantes normalistas de dos carreras en específico, alumnos de la Escuela Normal de Educación Preescolar y la Benemérita Escuela Normal de Coahuila para así contar con experiencias y expectativas variadas, así como maneras de ser y de pensar, no se asignó ninguna edad en especial, , se consideró un aspecto interesante dirigirse con personas de diferentes edades ya que si bien tienen un fin en común transmitir sus conocimientos a alguien más, no todos tienen las mismas estrategias y técnicas para impartir el conocimiento.

\section{Diseño de la investigación}

Dentro de este apartado se encontrará en primer lugar el enfoque de estudio, refiere a dar a conocer cuál será el enfoque que tomara esta investigación, el cual es cuantitativo, permitirá plantear un problema, así como descubrir y refinar preguntas que se presentarán a lo largo de dicha investigación. También se encontrará dentro de este capítulo, los alcances que la presente investigación llevara, lo que los estudios descriptivos pretenderán medir. Así como métodos de análisis para descubrir los resultados.

\section{Tipología de la investigació.}

La tipología es: correlacional asocia variables mediante un patrón predecible para un grupo o población.

Diseño del instrumento de investigación. 
El instrumento fue diseñado de manera simple y sencilla con variables acorde a los que se investiga y que fueran sencillas de responder, las partes del instrumento son: el encabezado donde se encuentran las instituciones que respaldan dicha investigación así como también la licenciatura de la cual proviene la aplicación del mismo, después se especifica el objetivo de la misma, siguen las instrucciones para responder el siguiente paso que son las preguntas generales de edad, genero, escuela y especialidad, luego se explica la forma de contestar seguido de un ejemplo breve, luego se presentan tres cuadros con los tres ejes y veinte variables significativas de los mismos y al final una frase de agradecimiento por haber cooperado con dicha investigación, la escala que se utilizo fue de Likert decimal, es decir del cero al diez donde el cero es ausencia de valor y diez es el máximo valor asignable según el criterio de los respondientes.

\section{Desarrollo y ejecución de la investigación}

Después de la recolección de datos se siguió con el vaciado de los mismos para poder hacer después la lectura de los resultados que se arrojaran después de haber utilizado los paquetes estadísticos habiendo realizado en la primera una análisis integracional de los datos para hacer un análisis factorial que consistían en relacionar todas las variables para poder relacionar unas con otras, y en el segundo paquete se realizaron análisis de regresión donde también se relacionaban una variables con otras para obtener los resultados que pudieran contestar al/los objetivos.

\section{Población.}

Para tal investigación la población fue de 213 alumnas de la Escuela Normal de Educación Preescolar y con 237 alumnos de la Benemérita Escuela Normal de Coahuila de ambas escuelas los alumnos pertenecían al tercer grado.

\section{Muestra.}

Del total de la población se tomó una muestra al azar de 70 sujetos, 44 de la ENEP y el resto (26) de la BENC.

\section{Marco teórico}

La sociedad en la que vivimos puede describirse como una sociedad que aprende, porque la clave del éxito es el conocimiento. Es una sociedad caracterizada por el intercambio de información y en la que el aprendizaje no es un proceso aislado. Así, la sociedad pone a disposición de los ciudadanos infinidad de recursos formativos, recursos que deben aprovecharse para que las personas puedan aprender las distintas profesiones y roles asuman la noción de aprender durante toda la vida.

A ello no escapa la profesión docente. Aprender a enseñar no es un acontecimiento ocasional ni de duración limitada, sino un proceso que dura toda la vida, comienza cuando quien sueña con hacer de la docencia su vocación, recibe su primera clase en una institución de formación docente y culmina cuando la vida lo coloca por última vez frente a sus alumnos (Chirinos Molero \& Padrón Añez, 2010).

El proceso de aprender a enseñar se asemeja a un río al que van confluyendo experiencias, conocimientos, situaciones, que se producen en contextos diferentes, en este caso, la universidad y las instituciones educativas que brindan sus espacios y recursos para que los estudiantes lleven a cabo sus prácticas; por tanto, se hace necesaria una cooperación más estrecha entre ambos contextos para que los sujetos implicados: estudiantes, maestros y profesores, aprendan continuamente $\mathrm{e}$ investiguen $\mathrm{y}$ construyan el conocimiento sobre la enseñanza. Visto así, el aprender a enseñar, como un proceso continuo y construido socialmente, apunta a transformaciones dinámicas en las prácticas educativas de los docentes

Transformaciones que deben responder a las inquietudes y necesidades particulares de cada uno y a las que sólo se llega cuestionando la propia práctica, reflexionando desde ella.

Por lo tanto, constituye un compromiso asumir la responsabilidad como formadores de docentes para que los estudiantes desarrollen durante su formación inicial la disposición reflexiva, así como la orientación hacia el aprendizaje permanente. Considerar que las prácticas profesionales docentes resulta el espacio idóneo para ello (Tallaferro, 2006). 
En las prácticas docentes se pretende que los estudiantes reflexionen sobre elementos relacionados con aspectos como: qué es un educador; conocimiento, comprensión y valoración de los niños; desarrollo de un pensamiento innovador; elaboración de un currículo eficaz; promoción de un ambiente de paz y armonía que permita rescatar los valores; creación de proyectos como alternativa extracurricular; incorporación de la familia a la institución educativa; uso de los recursos como apoyo a la labor educativa y la evaluación como alternativa eficaz para enriquecer los procesos de enseñanza y aprendizaje; por lo que su contenido está dirigido al alumno(a) que inicia esta experiencia académica como requisito fundamental para el logro de su meta profesional, tener una visión general del compromiso que conlleva la profesión docente, así como asumir una actitud positiva y abierta hacia el proceso educativo (Olivares Medina \& Mairena Vargas, 2002).

Las prácticas docentes en un sistema escolar de deficiente calidad, requieren adoptar un estilo diferente al que podría esperarse en sistemas escolares del mundo desarrollado, donde los practicantes tienen mayores posibilidades de ingresar a instituciones bien organizadas y encontrar modelos de excelencia profesional de los cuales aprender. Por el contrario, en nuestro contexto se enfrentan a situaciones de gestión escolar y de enseñanza deficientes a las que deben incorporarse sin intenciones de cambio. En este sentido, se podrían asumir los componentes de prácticas como una oportunidad para ofrecer y dar apoyo a las escuelas en su conjunto, con vistas a la actualización, la innovación y el cambio en asuntos pedagógicos y de gestión (Díaz, 2006).

Los docentes, como profesionales reflexivos, consideran a la pedagogía como la ciencia de la educación y no como la simple aplicación de conocimientos generados en otras disciplinas. Hernández, P. (2015). Los docentes reflexivos son profesionales críticos, practicantes permanentes de la reflexión intencional sobre su propia práctica como método de perfeccionamiento del ejercicio profesional. Se educan mientras mejoran su práctica y mejoran su práctica mientras educan.
Son profesionales transformadores que alimentan el deseo por encontrar nuevas soluciones y respuestas a los problemas del trabajo cotidiano, conscientes de su compromiso con los alumnos, con sus oportunidades para aprender, y con el futuro de la sociedad. Contrasta sus teorías y creencias mediante la aplicación para solucionar problemas prácticos, utiliza los resultados y la evaluación del proceso para descartar, asumir o mejorar las teorías (Fierro \& Carbajal, 2003).

El acercamiento a las complejidades de la realidad permitirá identificar los problemas prácticos para seleccionar contenidos teóricos de mayor valor en la formación de formadores. Como también poner a prueba las teorías que se enseñan y por supuesto describir y comprender la realidad, desarrollar conceptos basados en las observaciones, es decir, generar teorías propias fundamentadas en los contextos singulares, teorías sustantivas (Rodríguez Trujillo, 2007).

El objetivo explícito de la práctica reflexiva es el de lograr un conocimiento y una comprensión profunda que formará la base no sólo para considerar alternativas educativas, sino además para tomar acciones con el fin de mejorar continuamente la práctica a través de la carrera profesional que desarrolle el docente. Convertirse en un practicante de la reflexión significa crecer y expandirse de manera propia y abrirse a una mayor variedad de opciones posibles y respuestas a las situaciones del salón de clases. Construir el hábito de la práctica reflexiva permite a los practicantes desenvolverse con facilidad en el ambiente dinámico del aula de clases. Los que practican la reflexión se manejan en una espiral de aprendizaje permanente en el cual emergen los dilemas, iniciando constantemente un nuevo ciclo de observación, planificación, acción, reflexión, y adaptación, para luego continuar con el ciclo (Villalobos \& Cabrera de M., 2009).

Se puede sugerir la idea de la creación de talleres de experiencia en práctica docente, las cuales confirman las necesidades, por parte de los alumnos, de experimentar con otras opciones que tomen en cuenta los aspectos mencionados Op. Cit (2009). Estas propuestas surgen de quienes han asumido la formación como un intercambio crítico y reflexivo. Op. Cit (2009) 
El contenido de tales sugerencias proviene de observaciones y discusiones sobre lo que se considera problemas que caracterizan la vida del aula y sobre los que hay que tomar decisiones argumentadas, eficaces y eficientes. La concreción de esa problemática sugiere como actividades centrales:

La necesidad de considerar aplicaciones en extenso, es decir, durante la programación de un semestre o de un taller con las estrategias metodológicas seleccionadas, a manera de experimento durante la asistencia al taller, con el fin de evaluar su posible contribución en el tratamiento de la materia a la cual se va a aplicar.

El uso de variaciones de la Micro enseñanza que permitan recoger ejecuciones de unidades completas del taller, con el objeto de dar atención a los contenidos de la misma.

La experimentación con estrategias metodológicas recoge resultados benéficos, sobre todo en lo que respecta al trabajo de los alumnos. Esto, por cuanto el uso de cualquiera de esas estrategias exige que muchas de las actividades sean realizadas frente a grupo en los cuales los alumnos tendrán la oportunidad de experimentar lo que realizar los profesores y sentir la verdadera experiencia de la docencia: se podría decir que tienen que hacer exposiciones de contenidos; demostraciones de técnicas y equipos; discusiones organizadas y coordinadas por grupos de alumnos; para después en el taller realizar reportes verbales o escritos de experiencias recogidas de la práctica, búsquedas bibliográficas o visitas; progresos sobre investigaciones, individuales o en grupo, a través de seminarios (Pestana 2010).

La planeación es una etapa fundamental del desarrollo curricular porque se rige como el documento de referencia para las fases propias de la práctica. Por tanto, es muy importante elaborar el plan del currículo concentrando y analizando detenidamente toda la información disponible. La planeación estratégica permite clarificar e integrar con cierto orden los diferentes elementos que componen al currículo, el conjunto de conocimientos contemplados en el currículo no debería restringirse a los conocimientos técnicos en un área en específico, porque se requiere desarrollar un seguimiento de sucesos sociales y económicos (Ribes-Iñesta, 2008).
El diseño curricular es un proceso cuyo resultado es el documento que reúne un conjunto de elementos necesarios para lograr cierta formación en una institución de educativa: el currículo, la amplitud de esos elementos se extiende desde las razones que justifican la existencia y el funcionamiento del currículo hasta la estructura curricular, las características de los programas de las distintas unidades de aprendizaje que lo conforman, los docentes y administrativos que lo operan y los criterios que permiten su evaluación Op. Cit (2008).

La organización de todos los componentes de un currículo es un trabajo hasta cierto punto complejo, dada la integración de información en un documento racional, estructuralmente congruente y operativamente funcional (Aranda Barradas \& Salgado, 2005).

Los tipos de planeación observados aluden a distintos lapsos de tiempo considerados: anual, semanal, diaria, entre otros, o bien a los contenidos, como la planificación de una unidad de aprendizaje o lección (Rueda Beltrán, 2011).

Planteamiento sustentante: Las actuales reformas a la educación enfatizan el desarrollo de las habilidades para propiciar el integral de los estudiantes en el desarrollo de competencias que los preparen para su inserción en la actual sociedad y les apoyen en la habilidad de aprender a aprender a lo largo de la vida.

\section{Hipótesis.}

Los estudiantes de las escuelas normales creen que con la Reforma Educativa la calidad de la educación será mejor para todos.

La reforma educativa les aporta a los estudiantes normalistas las suficientes herramientas para crear un ambiente favorable dentro del aula.

La planeación docente es mejor que sea anual y que los padres tengan más acercamiento a la misma.

La práctica profesional dota a los estudiantes normalistas de suficientes conocimientos para saber ser un líder. 


\section{Resultados}

En el presente capitulo se muestran los resultados extraídos de los procesos estadísticos de análisis integracional: factorial y análisis de regresión. Donde se da una correlación entre todas las variables que dan respuesta a los objetivos.

\section{Pruebas de confiabilidad y consistencia interna}

Se realizó el análisis de confiabilidad dando como resultado el Alpha de Cronbach de .94 y un Alpha estandarizada también de .96 lo cual indica que dicha investigación es confiable y los resultados que se obtengan será importantes para los fines que se busca resaltar.

Nivel descriptivo: análisis de frecuencias y porcentajes (datos generales).

En la variable edad el $33 \%$ de los sujetos encuestados se encuentran en los 21 años, mientras que el $19 \%$ y el $18 \%$ corresponde a sujetos con edades de 20 y 22 años respectivamente y con los valores más bajos es decir un $11 \%$, un $8 \%$, un $7 \%$ y el $1 \%$ están en edades de 24, 23, 19 y 25 años respectivamente. En la variable género el $84 \%$ corresponde al género femenino y el $16 \%$ al género masculino. Lo cual indica que la mayor parte de los encuestados fueron mujeres.

En la variable escuela el $61 \%$ corresponden a la ENEP (Escuela Normal de Educación Preescolar) y al 38\% son de la BENC (Benemérita Escuela Normal de Coahuila).

En la variable especialidad el $44 \%$ son de la Licenciatura en Educación Preescolar y el $27 \%$ son de la Licenciatura en Educación Básica en español.

Nivel integracional: análisis factorial.

En la tabla se muestran los diferentes procedimientos integracionales, con rotación de factores Varimax normalizado, nivel de error $\mathrm{p} \leq$ 0.0001 y nivel de confianza del $99 \%$.

\begin{tabular}{|l|r|r|r|}
\hline Procedimiento & \multicolumn{1}{c}{$\begin{array}{c}\text { Numero } \\
\text { de } \\
\text { factores }\end{array}$} & \multicolumn{1}{c}{$\begin{array}{c}\text { Eigen } \\
\text { valor } \\
\text { acumulado }\end{array}$} & \multicolumn{1}{c|}{$\begin{array}{c}\text { Nivel de } \\
\text { explicación }\end{array}$} \\
\hline $\begin{array}{l}\text { Comm_multiple } \\
\text { R2 }\end{array}$ & 10 & 20.19 & 33.66 \\
\hline MINRES & 10 & 20.07 & 33.46 \\
\hline $\begin{array}{l}\text { Maximun } \\
\text { likelihood }\end{array}$ & 10 & 19.98 & 33.31 \\
\hline Centroid & 10 & 20.13 & 33.55 \\
\hline Principal axis & 9 & 20.04 & 33.40 \\
\hline
\end{tabular}

Tabla 1 Valor eigen acumulado de factorial

Se elige el procedimiento comunalidades de $\mathrm{R}^{2}$ por tener el mayor nivel de explicación de la variabilidad común y un número considerable de factores.

Análisis Integracional Factores.

\begin{tabular}{|l|r|}
\hline \multicolumn{2}{|c|}{ Planeación } \\
\hline Interacción maestro-alumno & 0.87 \\
\hline Interacción alumno-maestro & 0.80 \\
\hline Estrategias didácticas & 0.66 \\
\hline Técnicas de enseñanza & 0.64 \\
\hline Objetivos de aprendizaje & 0.55 \\
\hline Recursos didácticos & 0.55 \\
\hline
\end{tabular}

Tabla 2 Factor 1: planeación

En la Tabla 2 se muestran los factores que se relación para la planeación. Se infiere por lo tanto que los futuros docentes deben de plantear bien los objetivos de la enseñanza, definir las técnicas y estrategias para impartir una clase, saber utilizar los recursos adecuados para que se un aprendizaje significativo en los niños, además se necesita de una interacción tanto de los maestros hacia los alumnos, como de los alumnos a sus maestros para que este aprendizaje pueda ser reforzado en un momento dado.

\begin{tabular}{|l|c|}
\hline \multicolumn{2}{|c|}{ Habilidades de los estudiantes } \\
\hline Mercado laboral & 0.65 \\
\hline Productividad & 0.72 \\
\hline Obligaciones laborales & 0.87 \\
\hline Acatar ordenes & 0.73 \\
\hline Formación docente & 0.76 \\
\hline Aprendizaje en actividades laborales & 0.85 \\
\hline Empleados para trabajar en equipo & 0.67 \\
\hline Desarrollo personal & 0.61 \\
\hline Instituciones educativas & 0.75 \\
\hline Adquisición de habilidades sensoriales & 0.71 \\
\hline Uso de herramientas & 0.73 \\
\hline Adquisición de experiencia & 0.66 \\
\hline Función pedagógica & 0.57 \\
\hline Formación académica & 0.51 \\
\hline
\end{tabular}

Tabla 3 Factor 2: habilidades de los estudiantes 
En la Tabla 3 se muestran los factores que más se relacionan con el planteamiento de la investigación, es decir los alumnos deben de desarrollar habilidades propias de la profesión.

Por lo tanto, se infiere que estas habilidades harán que los estudiantes se desenvuelvan mejor dentro del área de trabajo que en este caso es frente el grupo, deben de saber a qué tipo de mercado laboral se insertaran para poder tener un mejor desarrollo tanto personal como laboral y así poder adquirir las habilidades necesarias para cumplir la función pedagógica que le ofrecerán al dar una formación académica de calidad a sus alumnos.

\begin{tabular}{|l|c|}
\hline \multicolumn{2}{|c|}{ Análisis de la planeación } \\
\hline Nuevos métodos de enseñanza & 0.85 \\
\hline Mejor desarrollo académico de los alumnos & 0.81 \\
\hline Nuevos procesos de evaluación & 0.60 \\
\hline Diagnóstico de necesidades educativas & 0.57 \\
\hline Necesidades educativas & 0.52 \\
\hline Actualización continua & 0.49 \\
\hline
\end{tabular}

Tabla3 Factor 3: análisis de la planeación

En la Tabla 3 se muestran los factores que se relacionan entre si para el análisis de la planeación. Por lo tanto, se infiere que se deben de definir nuevos métodos de enseñanza en base al conocimiento y diagnóstico de necesidades educativas con nuevos procesos de evaluación tanto para alumnos como para maestros, los cuales deben de estar en constante actualización para ayudar a los niños a tener un mejor desarrollo académico.

\begin{tabular}{|l|c|}
\hline \multicolumn{2}{|c|}{ Impartición de clase } \\
\hline Recursos didácticos & 0.61 \\
\hline Diseño de la planeación & 0.73 \\
\hline Evaluación del desempeño escolar & 0.83 \\
\hline
\end{tabular}

Tabla 4 Factor 4. Impartición de clase

En la Tabla 4 se aprecian los factores que se denominan impartición de clase, se infiere que para dar una clase se necesita del diseño de una planeación para definir los recursos necesarios para que los alumnos puedan asimilar mejor los contenidos, así como después de la clase llevar a cabo una evaluación del desempeño de los alumnos para poder observar si tienen algún avance o no.

\begin{tabular}{|l|l|}
\hline \multicolumn{1}{|c|}{ Cualidades de los estudiantes } & \\
\hline Saber ser líder & 0.51 \\
\hline Compromiso social & 0.72 \\
\hline Compromiso ético & 0.81 \\
\hline Formación académica & 0.58 \\
\hline Resolución de problemas & 0.64 \\
\hline
\end{tabular}

Tabla 5 Factor 5: cualidades de los estudiantes

En la Tabla 5 se muestran algunas cualidades que los futuros docentes deben de poseer. Se infiere que de acuerdo a la formación académica que reciban crearan en los estudiantes normalistas un compromiso ético y social el cual les ayudara a desenvolverse mejor y aprender a ser un líder dentro del salón de clase y resolver adecuadamente los posibles problemas que se lleguen a presentar.

\section{Factores que intervienen en la práctica}

\begin{tabular}{|l|c|}
\hline $\begin{array}{l}\text { Opinión de los padres de familia en los programas } \\
\text { educativos }\end{array}$ & 0.53 \\
\hline Entrenamiento & 0.66 \\
\hline Saber ser líder & 0.51 \\
\hline
\end{tabular}

Tabla 6 Factor 6: factores que intervienen en la práctica

En la tabla 6 se presentan las variables que se relacionan para definir los factores que intervienen en la práctica, por lo tanto, se infiere que la práctica profesional es un entrenamiento para el trabajo real, es decir cuando egresen, los preparan para desempeñar mejor el trabajo frente a grupo desarrollando un sentido de liderazgo propio de la carrera.

\section{El Estado y la Educación}

Que el Estado garantice los materiales educativos 0.72 \begin{tabular}{|l|l|}
\hline Conocimiento de los programas educativos & 0.68 \\
\hline
\end{tabular}

\begin{tabular}{|l|l|}
\hline Mejor infraestructura educativa & 0.64 \\
\hline Organizacon & 0.58 \\
\hline
\end{tabular}

\begin{tabular}{|l|l|}
\hline Organización escolar & 0.58 \\
\hline
\end{tabular}

\begin{tabular}{|l|l|}
\hline Nuevos proyectos educativos & 0.51 \\
\hline Directivos idoneos para su
\end{tabular}

Directivos idóneos para su puesto

0.49

Tabla 7 Factor 7: el Estado y la educación

En la Tabla 7 se observan las variables que se relacionan para definir el factor Estado y Educación. Los alumnos opinan que el Estado debe de garantizar los materiales adecuados para el mejor desarrollo académico de los alumnos, no solo didáctico y/o materiales para los maestros, sino tener una mejor infraestructura educativa como directivos idóneos para desempeñar mejor su papel además de que tengan un mejor conocimiento de los programas educativos y así poder llevar a cabo nuevos proyectos educativos en pro de la mejor calidad educativa y organización escolar. 


\begin{tabular}{|l|r|}
\hline \multicolumn{2}{|c|}{ Aprendizaje } \\
\hline Desarrollo integral del aprendizaje & 0.65 \\
\hline Aprendizaje significativo & 0.65 \\
\hline Análisis de la planeación & 0.63 \\
\hline Análisis de la evaluación & 0.63 \\
\hline Reforzamiento & 0.56 \\
\hline Diagnóstico del aprendizaje & 0.54 \\
\hline
\end{tabular}

Tabla 8 Factor 8: aprendizaje

En la Tabla 8 se aprecian las variables que formaron el factor aprendizaje. Se infiere que el desarrollo integral de aprendizaje de los alumnos se da a partir del aprendizaje significativo que se genere en ellos con el análisis de la planeación para después de aplicada la misma se de una evaluación del desempeño de los estudiantes y así hacer un análisis para definir si se da un reforzamiento y poder realizar un diagnóstico de los aprendido.

\begin{tabular}{|l|c|}
\hline \multicolumn{2}{|c|}{ Reforma Educativa } \\
\hline Calidad de la Educación & 0.73 \\
\hline Evaluaciones constantes & 0.54 \\
\hline Mejoramiento constante de los planes & 0.52 \\
\hline
\end{tabular}

Tabla 9 Factor 9: reforma Educativa

En la Tabla 9 se observan las variables que se relacionaron para formar el factor 9 Reforma educativa. Se infiere que para sé de una mejora en la calidad de la educación debe de haber evaluaciones constantes tanto a los directivos y maestros como a los alumnos y los planes y programas además de un mejoramiento contante en estos últimos para la actualización de la educación.

Objetivos de la reforma Educativa

\begin{tabular}{|l|c|}
\hline Relación con Evaluaciones Internacionales & 0.74 \\
\hline Aumento de la matrícula & 0.80 \\
\hline Máximo logro educativo de los alumnos & 0.52 \\
\hline
\end{tabular}

Tabla 10 Factor 10: objetivos de la Reforma Educativa

En la Tabla 10 se observan las variables que dieron nombre al factor 10 es decir, objetivos de la educación. Se infiere que con la Reforma Educativa se pretende que los resultados del aprendizaje se relacionen con evaluaciones internacionales, es decir que se haga un comparativo del aprendizaje entre varios países y se muestre el avance obtenido antes y después de la implementación de la Reforma, así como también se pretende el aumento de la matrícula en educación básica, es decir abarcar el mayor número de personas que reciban una educación de calidad y así puedan tener un máximo logro educativo.
Nivel relacional: análisis de regresión.

\begin{tabular}{|l|l|l|}
\multicolumn{1}{|c}{ Variable dependiente } & \multicolumn{1}{c|}{$\begin{array}{c}\text { Resolución de } \\
\text { problemas }\end{array}$} & $\begin{array}{l}\text { Rechaz } \\
\text { V la H0 }\end{array}$ \\
\hline Diagnóstico del aprendizaje & 0 & es \\
\hline Interacción alumno maestro & 0 & es \\
\hline $\begin{array}{l}\text { Mejor desarrollo académico de } \\
\text { los alumnos }\end{array}$ & 0 & es \\
\hline $\begin{array}{l}\text { Opinión de los padres de familia } \\
\text { en programas educativos }\end{array}$ & 0 & es \\
\hline
\end{tabular}

Tabla 11 Regresión múltiple

De este análisis las variables independientes que resultaron predictoras de la variable dependiente resolución de problemas, diagnóstico del aprendizaje, interacción alumnomaestro, mejor desarrollo académico de los alumnos y opinión de los padres de familia. Por lo que se infiere que los alumnos tienen un mejor desarrollo académico cuando los maestros llevan a cabo un diagnóstico del aprendizaje y se da una interacción positiva entre alumno-maestro, es decir que el alumno acude al maestro para que este le ayude o resuelva sus dudas hace que el alumnos se sientan más cómodo con los resultados de su aprendizaje, por otra parte refleja que los padres aporten una opinión acerca de los programas educativos de sus hijos para que así en conjunto toda la comunidad se dé una educación de calidad.

Regresión multivariada.

\begin{tabular}{|r|c|r|c|}
\hline Modelo & R2 & Cambio de R2 & Variables \\
\hline 10 & 0.764826 & 0.01318 & DEGLMOQBDN \\
\hline
\end{tabular}

Tabla 12 Regresión multivariada

De este análisis las variables independientes que resultaron predictoras de las variables dependientes diagnóstico del aprendizaje, interacción alumno-maestro, mejor desarrollo académico de los alumnos y opinión de los padres de familia son aumento de la matrícula, mejoramiento constante de los planes, que el Estado garantice los materiales educativos, maestros idóneos para su puesto, directivos idóneos para su puesto, opinión de los padres de familia en programas educativos, mejor desarrollo académico de los alumnos, diagnostico de aprendizaje, condiciones de aprendizaje y motivación que el docente debe de dar en clase. 
Por lo tanto se infiere que de acuerdo con la Reforma Educativa se pretende aumentar la matrícula de los niños que reciben una educación lo cual se dará a partir de que el Estado garantice los recursos educativos necesarios para que exista un mejoramiento constante de los planes además de que maestros y directivos sean idóneos para ocupar el puesto que se les asigne, es decir que sepan cómo llevar a cabo a su trabajo y siempre con miras a mejorar el desarrollo académico de los alumnos, como otorgando mejores condiciones de aprendizaje donde el niño se desenvuelva satisfactoriamente aunado a la motivación que el docente presente al momento de estar frente a grupo, los resultados del aprendizaje se harán mediante un diagnostico donde el niño refleje los avances más significativos a lo largo de su estancia en la escuela, otro factor que podría ayudar a mejorar la calidad de la educación seria la opinión de los padres en cuanto a los programas educativos, ellos también podrían hacer un diagnóstico del aprendizaje de sus hijos, en que han mejorado, que les falta o que no han podido desarrollar y así poder hacer cambios en la planeación del docente donde intente otras posibilidades de enseñanza o refuerce las ya dadas.

Todas las posibles regresiones.

En este procesamiento estadístico, se obtuvo el mejor modelo de regresión que da la mejor explicación al planteamiento sustentante. En la siguiente tabla se muestran 15 variables que conforman el modelo de regresión, es decir el mejor especificado.

\section{Número de modelo $\quad$ CP $\quad$ Modelo}

$$
\begin{array}{l|l|l|}
5 & 6.716311 & \text { FGJLS }
\end{array}
$$

Tabla 13 Todas las posibles regresiones

De acuerdo a los resultados obtenidos y presentados en la tabla seis el mejor modelo para llevarlo a cabo en los alumnos está integrado por 5 variables independientes que predicen a la variable dependiente se adapten a la resolución de problemas. Por lo tanto, se infiere que se deben de plantear bien los objetivos de una planeación anual para que aunado a las estrategias que presente el docente y la motivación que tenga al estar frente al grupo se dé un mejor desarrollo académico.

\section{Conclusiones}

De acuerdo con la Reforma Educativa el impacto que ha causado tanto en la planeación didáctica como en la práctica profesional es que por medio de la Reforma se pretende aumentar la matrícula de los niños que reciben una educación lo cual se dará a partir de que el Estado garantice los recursos educativos necesarios para que exista un mejoramiento constante de los planes además de que maestros y directivos sean idóneos para ocupar el puesto que se les asigne, es decir que sepan cómo llevar a cabo a su trabajo y siempre con miras a mejor el desarrollo académico de los alumnos, como otorgando mejores condiciones de aprendizaje donde el niño se desenvuelva satisfactoriamente aunado a la motivación que el docente presente al momento de estar frente a grupo, los resultados del aprendizaje se harán mediante un diagnostico donde el niño refleje los avances más significativos a lo largo de su estancia en la escuela, otro factor que podría ayudar a mejorar la calidad de la educación seria la opinión de los padres en cuanto a los programas educativos, ellos también podrían hacer un diagnóstico del aprendizaje de sus hijos, en que han mejorado, que les falta o que no han podido desarrollar y así poder hacer cambios en la planeación del docente donde intente otras posibilidades de enseñanza o refuerce las ya dadas.

\section{Discusión}

Visto así, el aprender a enseñar, como un proceso continuo y construido socialmente, apunta a transformaciones dinámicas en las prácticas educativas de los docentes. Transformaciones que deben responder a las inquietudes y necesidades particulares de cada uno y a las que sólo se llega cuestionando la propia práctica, reflexionando desde ella.

Por lo tanto, constituye un compromiso asumir la responsabilidad como formadores de docentes para que los estudiantes desarrollen durante su formación inicial la disposición reflexiva, así como la orientación hacia el aprendizaje permanente. Considerar que las prácticas profesionales docentes resulta el espacio idóneo para ello (Tallaferro, 2006). 
De acuerdo con el autor en los resultados se refleja que los estudiantes deben de adquirir ciertas habilidades que harán que su trabajo frente a grupo sea el mejor y que los conocimientos que transmiten no solo sean pasajeros, sino que les deje a sus alumnos las habilidades necesarias para poder desenvolverse en el mundo actual.

En las prácticas docentes se pretende que los estudiantes reflexionen sobre elementos relacionados con aspectos como: qué es un educador; conocimiento, comprensión y valoración de los niños; desarrollo de un pensamiento innovador; elaboración de un currículo eficaz; promoción de un ambiente de paz y armonía que permita rescatar los valores; creación de proyectos como alternativa extracurricular; incorporación de la familia a la institución educativa; uso de los recursos como apoyo a la labor educativa y la evaluación como alternativa eficaz para enriquecer los procesos de enseñanza y aprendizaje; por lo que su contenido está dirigido al alumno(a) que inicia esta experiencia académica como requisito fundamental para el logro de su meta profesional, tener una visión general del compromiso que conlleva la profesión docente, así como asumir una actitud positiva y abierta hacia el proceso educativo (Olivares Medina \& Mairena Vargas, 2002). Los futuros docentes deben de asumir un compromiso social y ético para que desarrollen en los niños ese sentimiento por aprender y que puedan desarrollar de mejor manera sus habilidades.

Las prácticas docentes en un sistema escolar de deficiente calidad, requieren adoptar un estilo diferente al que podría esperarse en sistemas escolares del mundo desarrollado, donde los practicantes tienen mayores posibilidades de ingresar a instituciones bien organizadas y encontrar modelos de excelencia profesional de los cuales aprender. Por el contrario, en nuestro contexto se enfrentan a situaciones de gestión escolar y de enseñanza deficientes a las que deben incorporarse sin intenciones de cambio. En este sentido, se podrían asumir los componentes de prácticas como una oportunidad para ofrecer y dar apoyo a las escuelas en su conjunto, con vistas a la actualización, la innovación y el cambio en asuntos pedagógicos y de gestión (Díaz, 2006).
De acuerdo con el autor el actual sistema educativo superior formar de nuevos docentes presenta deficiencias muy marcadas que hacen que se pierdan los verdaderos objetivos de la educación que es aprender, pero aprender con calidad, es decir que los maestros asuman su rol de buenos formadores y doten de experiencias enriquecedoras a sus alumnos para que puedan desenvolverse en un futuro.

\section{Propuesta}

Como propuesta se tiene impartir un diplomado acerca de la nueva Reforma Educativa mexicana, ya que los futuros docentes conocen muy poco acerca de esta nueva reforma con el curso se pretende actualizar a los alumnos normalistas para que conozcan los objetivos y los alcances de dicha reforma y cómo impacta tanto en su formación académica como en la formación de sus futuros alumnos.

\section{Referencias}

Ander-Egg, Ezequiel. (2004). Introducción a la Planificación (cuarta edición). Editorial Lumen. Argentina.

Aranda Barradas, J. S., \& Salgado, M. E. (Mayojunio de 2005). El diseño curricular y la planeación estrategica. (I. P. Nacional, Ed.) Innovación educativa, 5(26), 25-35.

Arnaz, José. (1981). La planificación curricular (segunda edición). Editorial Trillas. México.

Bateman, Thomas y Snell, Scout A. (2004). Administración una Ventaja Competitiva (cuarta edición). Editorial Mc graw Hill. México.

Chirinos Molero, N., \& Padrón Añez, E. (septiembre.diciembre de 2010). La eficiencia docente en la práctica educativa. Revista de ciencias sociales, 16(3), 481-492.

Correa de Molina, Cecilia. (2004). Currículo dialógico, sistémico e interdisciplinar. Subjetividad y Desarrollo Humano (segunda edición). Cooperativa Editorial Magisterio Transversales. Colombia. 
Devalle de Rendo A. (2010). La formación docente. Según las representaciones de los futuros maestros. Buenos Aires: Lugar Editorial.Díaz, Q. (2006). Formación docente, Práctica pedagógica y saber pedagógico. Revista de educación Laurus, 12, 88-103.

Fierro, C., \& Carbajal, P. (febreo-julio de 2009). El docente y los valores desde su práctica. Sinéctica(22), 3-11.

Hernández, P. (2015). Diseñar y enseñar. Teoría y técnicas de la programación y del proyecto docente. Madrid: Narcea.

Lafrancesco, Giovanni. (2004). Currículo y Plan de Estudios. Estructura y Planeamiento (cuarta edición). Cooperativa Editorial Magisterio. Colombia.

Olivares Medina, M., \& Mairena Vargas, N. (Mayo de 2002). Retos didácticos en la práctica docente. Intersedes: revista de las sedes regionales, 3(5), 91-102.

Pestana, N. (julio-septiembre de 2010). Sobre formación docente y su concresión en la práctica. Educere, 4(10), 35-38.

Plan y programas de estudio 2011. México: SEP. SEP. (2011b). La formación científica en el perfil de egreso del alumno de educación básica. México: SEP.

Ribes-Iñesta, E. (Diciembre de 2008). Educación básica, desarrollo psicológico y planeación de competencias. (S. M. Psicología, Ed.) Revista Mexicana de Psicología, 25(2), 193-207.

Rodríguez Trujillo, N. (Diciembre de 2007). Prácticas docentes y mejora de la escuela. REICE. Revista Iberoamericana sobre calidad, eficacia y cambio en educación, 5(5), 21-26.

Rueda Beltrán, M. (2011). La investigación sobre la planeación educativa. (I. d. Educación, Ed.) Perfiles educativos, 33(131), 3-6

Rueda, C. (2016). Programa de Maestría en enseñanza de las ciencias. México: ENSVTSEIEM.

Santillana. (2003). Diccionario de las Ciencias de la Educación. España: Santillana. SEP. (2011a).
Tallaferro, D. (abril-junio de 2006). La formación para la práctica reflexiva en las prácticas profesionales docentes. Educere, 10(33),289- 273

UNESCO. (2009). La UNESCO Retos Mundiales. Recuperado de http://unesdoc.unesco.org/images/0014/001473/ 147330s.pdf Zabala, A. (2010). La práctica educativa. Cómo enseñar. España: Graó.

Villalobos, J., \& Cabrera de M., C. (enero-junio de 2009). Los docentes y su necesidad de ejercer una practica reflexiva. Revista de Teoría y Didáctica de las Ciencias Sociales(14) 139-166 